\title{
Design and Implementation of Optical Fiber Communication System for Field Area Networks of Smart Grid
}

\author{
Zhongwei Sun \\ School of Electrical \& Electronic Engineering North China Electric Power University, Beijing, China \\ zwsun@ncepu.edu.cn
}

\begin{abstract}
The smart grid communication infrastructure will emerge from the interconnection of a large number of small-scale networks organized into a hierarchical architecture of Home Area Networks (HANs), Feighborhood Area Networks (FANs) and Wide Area Networks (WANs). FANs form the communication facility for the electricity distribution systems and act as a bridge between customer premises and substations with collectors, access points and data concentrators. However, the existing communications network is insufficient for the FANs due to the factor that it does not cover the distribution side. This paper focuses on design and implementation of communication system for FANs in the smart grid. The communication infrastructure using Ethernet Passive Optical Networks (EPONs) is ultilized. Based on the power system applications operating in the distribution domain, communication system for both field based application and customer based application are presented

Index Terms - Smart grid, Field area networks (FANs), Ethernet Passive Optical Networks (EPONs).
\end{abstract}

\section{Introduction}

Today, The electricity is generated and distributed in a hierarchical power grid that has three distinct subsystems: generation, transmission, and distribution. The power plants generate electricity and then step-up transformers at the transmission substations convert it into high voltage electricity for long-distance transmission on the grid. At the distribution substations, this high voltage electricity is converted into medium voltage and transported over the distribution grid to the end users. Before entering the end user premises, the medium voltage is converted into low voltage. This basic flow of electric power in power grid has remained unchanged for a little over a century. It is increasingly outdated and overburdened, leading to costly blackouts and burnouts. For this and various other reasons, transformation efforts are underway to make the current electrical grid smarter[1,2].

A smart grid (SG), also called intelligent grid, is an enhancement of the 20th century power grid. The traditional power grids are generally used to carry power from a few central generators to a large number of users or customers. In contrast, the SG uses two-way flows of electricity and information to create an automated and distributed advanced energy delivery network. By utilizing modern information technologies, the SG is apable of delivering power in more efficient ways and responding to wide ranging conditions and events. More specifically, the $\mathrm{SG}$ can be regarded as an electric system that uses information, two-way, cyber-secure communication technologies, and computational intelligence in an integrated fashion across electricity generation, transmission, substations, distribution and consumption to achieve a system that is clean, safe, secure, reliable, resilient, efficient, and sustainable [3-6].

The smart grid is striving to utilize and coordinate various generation and production mechanisms. It consists of many different types of networks and has a tiered architecture to supply energy to consumers. As one of the enabling technologies, a fast, reliable and secure communication network plays a vital role in the SG. The network is required to connect the magnitude of electric devices in distributed locations and exchange their status information and control instructions. However, the existing communications network is inadequate, inflexible, and expensive. The inadequacy stems mainly from the following factors: first, the existing communications network only covers generation and transmission segments. It does not cover the distribution side where the major changes are expected to occur. Second, the capacity and speed of the installed communications network are inadequate to accommodate the future capacity growth and speed requirements of SG applications [7-9].

A robust communication infrastructure, which consists of different network components, such as Home Area Networks (HANs), Feighborhood Area Networks (FANs) and Wide Area Networks (WANs), is the touchstone of a smart grid. However, the existing communications network is insufficient due to the factor that it does not cover the distribution side where the major changes are expected to occur. Hence, this paper focuses on design aspect of communication architecture for FANs, and EPON-based FAN communication systems for both field based application and customer based application are discussed.

\section{Smart grid communications networks}

The SG is a modern electric power grid infrastructure for improved efficiency, reliability and safety, with smooth integration of renewable and alternative energy sources, through automated control and modern communications technologies. The communication infrastructure in smart grid must support the expected smart grid functionalities and meet the performance requirements. As the infrastructure connects an enormous number of electric devices and manages the 
complicated device communications, it is constructed in a hierarchical architecture with interconnected individual subnetworks and each taking responsibility of separate geographical regions. An illustrative example of this architecture is shown in Fig. 1

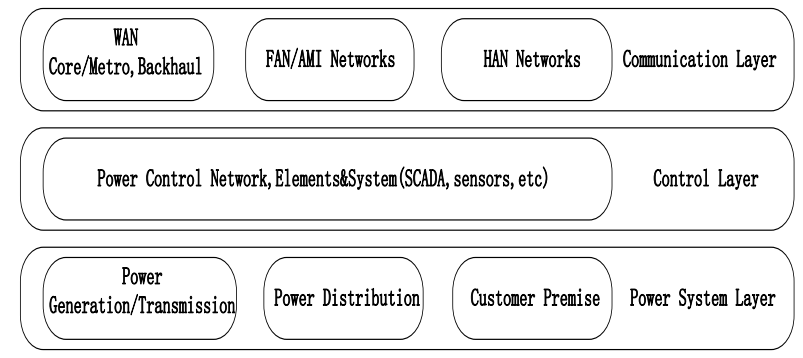

Fig. 1 The Smart Grid Communications Framework

Home area networks (HANs) are needed in the customer domain to implement monitoring and control of smart devices in customer premises and to implement new functionalities like DR and AMI. HANs also enable home automation networks for monitoring and control applications for user comfort, efficient home management, and DR application.

Field Area Network (FAN) can be described as the communication network for power distribution areas and includes distribution automation and control devices communicating over networks between individual service connections and backhaul points to the electric utilities. It acts as a bridge between customer premises and substations with collectors, access points and data concentrators. Intelligent nodes are deployed between customer premises and substations to collect and control the data from surrounding data points.

Wide Area Network (WAN) provides communications link between the grid and core utility systems. WAN comprises two types of networks:Core and Backhaul. While the Core Network is used to connect metro network of the utility and substations, the Backhaul Network is used to connect FAN to the core network.

\section{Available Art Communication Network for FANs}

A. FANs Communication requirements

FANs communication requirements aredriven by business functional requirements which may includethe following aspects $[10,11]$ :

- Feeder status monitoring

- Feeder voltage quality monitoring

- Reactive power monitoring (capacitor bank monitoring)

- Managing reactive compensation (capacitor bank switching)

- Feeder switch control

- Feeder sectionalizer and recloser control

- Supervisory control of feeder fault isolation schemes

- Provide communication channels for fault isolation schemes
- Monitor customer power quality

- Read customer meters for total usage

- Read customer time-of-use usage

- Control end-use loads according to predetermined schedules

- Control end-use loads according to system conditions, such as peak load periods

\section{B. Supporting Communication Technologies}

Many communication technologies can be used for FANs of the smart grid, The communication technologies evaluated for FANs are: (i) Power line communication, (ii) Wireline communication, (iii) Wireless communication.

1) Power Line Communication: The power lines are mainly used for electrical power transmissions, but they can also be utilized for data transmissions. Power line communication is a technique that uses the existing power lines to transmit high speed data signals from one device to the other. The power line communication systems operate by sending modulated carrier signals on the power transmission wires. Typically data signals cannot propagate through transformers and hence the power line communication is limited within each line segment between transformers. Data rates on power lines vary from a few hundred of bits per second to millions of bits per second, in a reverse proportional relation to the power line distance.

2) Wireline Network: Dedicated wireline cables can be used to construct data communication networks that are separate from the electrical power lines. These dedicated networks require extra investment on the cable deployment, but they can offer higher communication capacity and shorter communication delay. Depending on the transmission medium used, the wireline networks include SONET/SDH, Ethernet, coaxial cable access network, and Passive Optical Networks (PONs).

3) Wireless Network. Advancement in wireless networking technology has enabled us to connect devices in a wireless way, eliminating the installation of wirelines. IEEE 802.11s, RF Mesh, Worldwide Interoperability for Microwave Access (WiMAX) and cellular standards, such as 3G, 4G, and LTE, are some of the stronger candidates for the FANs.

\section{FAN communication network using EPON}

\section{A. $E P O N$}

The Ethernet Passive Optical Network (EPON) is a PON in combination with Ethernet. The EPON consists of one Optical Line Termination (OLT), multiple Optical Network Units (ONUs), and an Optical Distribution Network (ODN) with passive optical components. All transmission in the EPON are performed between an OLT and all ONUs connected with the OLT, as shown in Fig. 2. In the downstream direction, the EPON is a point-to-multipoint network, and an OLT broadcasts the information to all ONUs. In the upstream direction, the EPON is multipoint-to-point network, the whole available upstream channel bandwidth is divided into transmission units (typically termed slots) using 
the Time Division Multiplexing (TDM) technique, which can be assigned to the active ONUs [12].

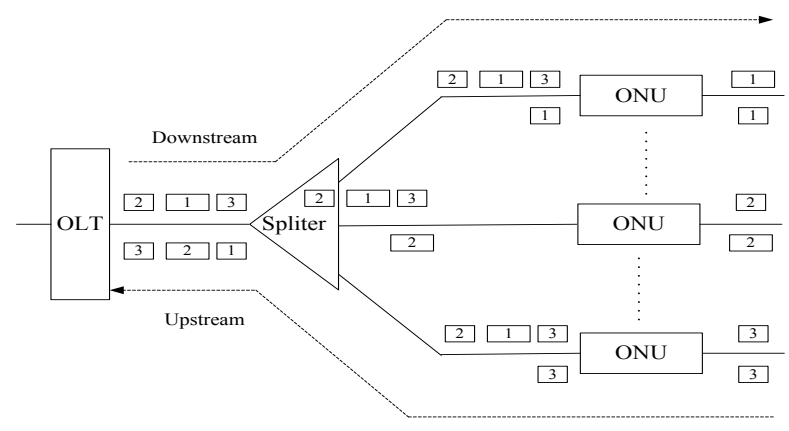

Fig. 2. The principle of EPON

There are several multipoint topologies suitable for the EPON, including tree, tree-and-branch, ring, and bus, as shown in Fig. 3 [13,14].

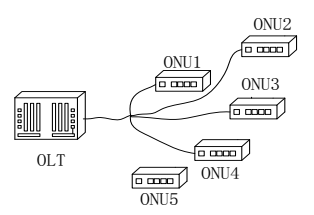

(a) Tree topology

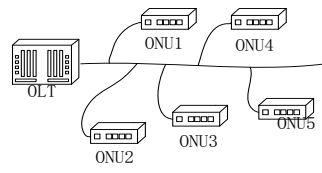

(b) Bus topology

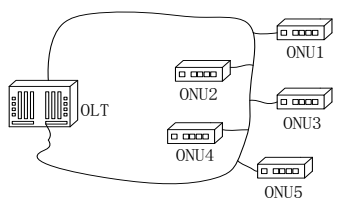

(c) Ring topology

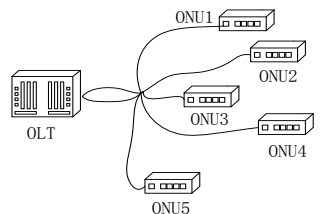

(d) Tree with redundant trunk
Fig. 3. Typical EPON topology structure.

\section{B. The proposed Communication network}

Field area networks form the communication facility for the electricity distribution systems. The power system applications operating in the distribution domain utilize field area networks to share and exchange information. These applications can be categorized as either field based or customer based. For field based applications, feeder automation is basic functional requirement of Distribution Automation System (DAS), where hand-in-hand loop distribution line connection is the widely used in urban distribution network. Fig.4 shows the EPON communication network for feeder automation, where various monitoring, control and protection funtionalities can be realized. As shown in the figure, the bus topology is adopted, and a redundant EPON communication structure is employed, due to the high reliability requirement of DAS. When the working EPON communication link breaks down, the backup EPON communication link will be activated and the backup EPON communication link will be used.

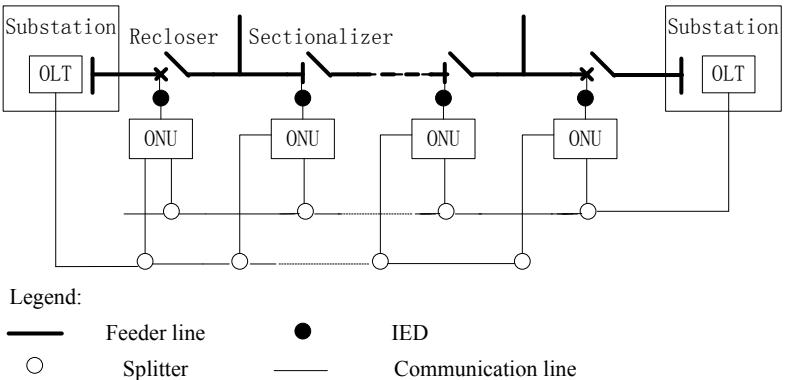

Fig. 4. EPON system for feeder automation

For the customer based applications, the interaction between the customer domain and other domains is achieved through Advanced Metering Infrastructure (AMI). Generally speaking, utility companies have been considering both wired and wireless communication technologies for building AMI networks. However, wired technologies are considered superior to wireless technologies in terms of reliability, security, and bandwidth because cables are easier to protect from interference and eavesdroppers. Fig.5 illustrates the possible types of connectivity using EPON communication network within AMI, where the tree topology is adopted for the EPON.

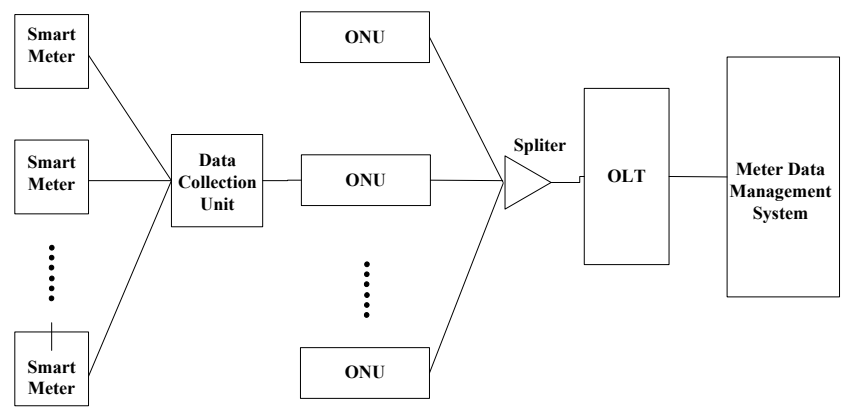

Fig. 5. EPON system for AMI

\section{Conclusions}

A robust communication infrastructure is the touchstone of a smart grid. In this paper, we have discussed the communication infrastructure of field area network using Ethernet Passive Optical Networks (EPON). Based on the power system applications operating in the distribution domain, communication system for both field based application and customer based application are presented.

\section{References}

[1] N.I. of Standards, T. (NIST), NIST framework and roadmap for smart grid interoperability standards, Release 1.0, 2010.

[2] R.H. Katz, D.E. Culler, S. Sanders, S. Alspaugh, Y. Chen, S. Dawson Haggerty, P. Dutta, M. He, X. Jiang, L. Keys, A. Krioukov, K. Lutz, J. Ortiz, P. Mohan, E. Reutzel, J. Taneja, J. Hsu, S. Shankar: An information-centric energy infrastructure: the berkeley view, Sustainable Computing: Informatics and Systems.Vol.1, no. 2, pp.7-22, 2011.

[3] X. Fang, S. Misra, G. Xue, D. Yang: Smart grid - the new and improved power grid: a survey. IEEE Communications Surveys Tutorials. Vol. 14,no.4, pp.944-980, 2011. 
[4] V. Cagri Gungor, Dilan Sahin, Taskin Kocak, Salih Ergut, Concettina Buccella, Carlo Cecati, and Gerhard P. Hancke: A Survey on Smart Grid Potential Applications and Communication Requirements. IEEE Trans. On Industrial Informatics, Vol. 9,no.1, pp.28-42, 2013.

[5] J. Gao, Y. Xiao, J. Liu, W. Liang, C.P. Chen: A survey of communication/networking in smart grids, Future Generation Computer Systems. Vol. 28, no.5, pp.391-404, 2012.

[6] Y. Yan, Y. Qian, H. Sharif, and D. Tipper: A survey on smart grid communication infrastructures: Motivations, requirements and challenges. IEEE Commun. Surveys \& Tutorials, vol., no. 99, pp.1-16

[7] Wenye Wang, Yi Xu, Mohit Khanna: A survey on the communication architectures in smart grid. Computer Networks. Vol. 55, pp.3604-3629, 2011.

[8] N. Saputro et al., A survey of routing protocols for smart grid communications, Computer Networks, Vol.56, pp.2742-2771, 2012.

[9] Reduan H. Khan, Jamil Y. Khan: A comprehensive review of the application characteristics and traffic requirements of a smart grid communications network. Computer Networks, Vol.57, pp. 825-845, 2013.

[10] D.E. Nordell: Communication systems for distribution automation. IEEE PowerTech, Bucharest, pp.1-8, 2009.

[11] D.M.Staszesky, D.Craig, C.Befus, "Advanced feeder automation is here", IEEE Power \& Energy Magazine, vol.20, pp. 56-63, 2005.

[12] Hiroaki Mukai, Yoshifumi Hotta, Tetsuya Yokotani, Akira Takahashi, Kiyoshi Shimokasa: PON with automatic protection switching for high reliable communication. Optical Switching and Networking, Vol.6, pp.163-17, 2009.

[13] Glen Kramer, Gerry Pesavento: Ethernet Passive Optical Network (EPON): Building a Next-Generation Optical Access Network. IEEE Communications Magazine, Vol.7, no.2, pp.66-73, 2002.

[14] Zhong-wei Sun, Qingrui Guo, Yanin Ma,Fengjie Sun: Communication system for distribution automation using EPON. in: Proceedings of the International conference on computational intelligence and software engineering, (2009). 